\title{
Steady state for a predator-prey cross-diffusion system with the Beddington-DeAngelis and Tanner functional response
}

\section{Demou Luo ${ }^{1 *}$ (D)}

"Correspondence:

223787092@qq.com

${ }^{1}$ School of Mathematics, Sun

Yat-sen University, Xingang West Road, 510275 Guangzhou, P.R. China

\begin{abstract}
The main goal of this paper is investigating the existence of nonconstant positive steady states of a linear prey-predator cross-diffusion system with Beddington-DeAngelis and Tanner functional response. An analytical method and fixed point index theory plays a significant role in our main proofs.
\end{abstract}

Keywords: Beddington-DeAngelis and Tanner functional response; Prey-predator; Cross-diffusion; Steady state

\section{Introduction}

We propose and study a predator-prey cross-diffusion system with the BeddingtonDeAngelis and Tanner functional response

$$
\begin{cases}\frac{\partial u}{\partial t}-d_{1} \Delta u-d_{2} \Delta v=u\left(a-u-\frac{v}{1+\alpha u+\beta v}\right), & x \in \Omega, t>0, \\ \frac{\partial v}{\partial t}+d_{3} \Delta u-d_{4} \Delta v=v\left(b-\frac{v}{\gamma u}\right), & x \in \Omega, t>0, \\ \frac{\partial u}{\partial v}=\frac{\partial v}{\partial v}=0, & x \in \partial \Omega, t>0, \\ u(x, 0)=u_{0}(x)>0, \quad v(x, 0)=v_{0}(x) \geq(\not \equiv) 0, & x \in \Omega,\end{cases}
$$

where $a, b, \alpha, \beta, \gamma$ are positive constants. Here $u$ and $v$ stand for the population densities of the prey and predator, respectively. The bounded domain $\Omega \in \mathbb{R}^{n}(n \geq 1)$ possesses a smooth boundary $\partial \Omega ; v$ is the outward directional derivative normal to $\partial \Omega$. The diffusion rates are described by $d_{1}>0, d_{2}>0, d_{3}>0$ and $d_{4}>0 ; a$ and $b$ are the death rate of the predator and the intrinsic growth rate of the prey, respectively. The smooth functions $u_{0}$ and $v_{0}$ on $\Omega$ represent the initial population densities of prey and predator. The corresponding ordinary differential system (ODS) of (1.1) is performed as

$$
\left\{\begin{array}{l}
\frac{d u}{d t}=u\left(a-u-\frac{v}{1+\alpha u+\beta v}\right), \\
\frac{d v}{d t}=v\left(b-\frac{v}{\gamma u}\right),
\end{array}\right.
$$

(0) The Author(s) 2020. This article is licensed under a Creative Commons Attribution 4.0 International License, which permits use sharing, adaptation, distribution and reproduction in any medium or format, as long as you give appropriate credit to the original author(s) and the source, provide a link to the Creative Commons licence, and indicate if changes were made. The images or other third party material in this article are included in the article's Creative Commons licence, unless indicated otherwise in a credit line to the material. If material is not included in the article's Creative Commons licence and your intended use is not permitted by statutory regulation or exceeds the permitted use, you will need to obtain permission directly from the copyright holder. To view a copy of this licence, visit http://creativecommons.org/licenses/by/4.0/. 
which was introduced by Luo [1] in recent years, and the globally asymptotical stability of (1.2) has been discussed by constructing a feasible Lyapunov function. Recently, with rapid development of biotechnology, we need to accurately illustrate the interaction between prey and predator in a real ecological environment such as hare and lynx, sparrow hawk and sparrow, spider mite and mite, and so on. Such interesting natural phenomena as the existence and global stability of interior periodic solutions and the global stability of reaction-diffusion system have been described by this ODE model [1] or the corresponding PDE system [2]. By utilizing the iteration method, Liu and Li [2] proved the globally asymptotic stability of the corresponding parabolic PDE system.

The impact of prey-taxis leads to the linear cross-diffusion in model (1.1), which means that the prey attraction to the predator may give rise to the migration of predator following the gradient of the density function of prey. By utilizing $-d_{3} \Delta u$ to describe this impact we will incorporate $d_{2} \Delta v$ into the first equation of system (1.1) to indicate the repelling effect from predator to prey. The message about the location of predator can guide the prey escape from them (see [3-5]).

The Beddington-DeAngelis functional response $\frac{u v}{1+\alpha u+\beta v}$ was firstly discovered by Beddington [6] and DeAngelis et al. [7] and intensively investigated by many researchers in mathematics, ecology, and biology (see $[1,2,8,9]$ ). This functional response, in the denominator, includes an additional term $\beta v$ compared with Holling-II functional response. This term illustrates the interaction effect among predators and provides a more general situation to describe the interaction between prey and predator.

As far as we can survey, this is the first paper studying the steady state for a predatorprey cross-diffusion system with the Beddington-DeAngelis and Tanner functional response. For notational simplicity, we introduce some notations used in the rest of this paper.

\section{Notation}

(1) We denote the eigenvalues sequence by $0=\lambda_{0} \leq \lambda_{1} \leq \cdots \leq \lambda_{k} \leq \cdots$ for the elliptic operator $\Delta$ on $\Omega$ with homogeneous Neumann boundary condition;

(2) The multiplicity of the eigenvalue $\lambda_{k}$ is denoted by $\mathcal{M}_{k}$;

(3) The corresponding normalized eigenfunctions of $\lambda_{k}$ are represented by the set $\left\{\mathcal{E}_{k j}: 1 \leq j \leq \mathcal{M}_{k}\right\}$

(4) $\mathcal{A}=C_{v}^{1}(\bar{\Omega}) \oplus C_{v}^{1}(\bar{\Omega})$, where $C_{v}^{1}(\bar{\Omega}) \triangleq\left\{\Phi \in C^{1}(\bar{\Omega}): \frac{\partial \Phi}{\partial v}=0\right.$ on $\left.\partial \Omega\right\}$;

(5) $\mathcal{D}=\mathcal{D}_{u} \oplus \mathcal{D}_{v}$, where $\mathcal{D}_{u} \triangleq\left\{\Phi \in C_{v}^{1}(\bar{\Omega}): \Phi<a\right.$ in $\left.\bar{\Omega}\right\}$ and $\mathcal{D}_{v} \triangleq\left\{\Phi \in C_{v}^{1}(\bar{\Omega}): \Phi<a b \gamma\right.$ in $\left.\bar{\Omega}\right\}$

(6) $\mathcal{Q}=\mathcal{S} \oplus \mathcal{S}$, where $\mathcal{S} \triangleq\left\{\Phi \in C_{v}^{1}(\bar{\Omega}): \Phi(x) \geq 0, x \in \bar{\Omega}\right\}$;

(7) $\mathcal{D}^{\prime}=\mathcal{D} \cap \mathcal{Q}$.

The rest part of this paper is organized as follows. In Sect. 2, we provide the study of the local stability of semitrivial or interior equilibrium of model (1.1). In Sect. 3, by utilizing the fixed point index theory we investigate the existence of the nonconstant positive steady state of system (1.1). Finally, in Sect. 4, we offer some conclusions on our main theoretical results. 


\section{Local stability}

It is obvious via a direct calculation that model (1.1) possesses one semitrivial equilibrium $(a, 0)$ and one unique interior equilibrium $\left(u^{*}, v^{*}\right)$, where

$$
u^{*}=\frac{[a \alpha-1+b \gamma(a \beta-1)]+\sqrt{[a \alpha-1+b \gamma(a \beta-1)]^{2}+4 a(\alpha+b \beta \gamma)}}{2(\alpha+b \beta \gamma)}, \quad v^{*}=b \gamma u^{*}
$$

We discuss the local stability of the semitrivial equilibrium $(a, 0)$ and the interior equilibrium $\left(u^{*}, v^{*}\right)$ of system (1.1) as follows.

\section{Lemma 2.1}

(1) When $a<b,(a, 0)$ is unstable.

(2) When $\frac{b \gamma u^{*}\left[1+(2 \alpha+b \beta \gamma) u^{*}\right]}{\left[1+(\alpha+b \beta \gamma) u^{*}\right]^{2}}<a+b$ and $a>\frac{b \gamma u^{*}(\alpha+b \beta \gamma) u^{*}}{\left[1+(\alpha+b \beta \gamma) u^{*}\right]^{2}}$, then the interior equilibrium $\left(u^{*}, v^{*}\right)$ is locally asymptotically stable.

(3) When $a+b<\frac{b \gamma u^{*}\left[1+(2 \alpha+b \beta \gamma) u^{*}\right]}{\left[1+(\alpha+b \beta \gamma) u^{*}\right]^{2}}$, then the interior equilibrium $\left(u^{*}, v^{*}\right)$ is unstable.

Proof At a constant solution $E^{*}=(u, v)$, the linearized issue of model (1.1) can be proposed by

$$
\left(\begin{array}{l}
\frac{\partial u}{\partial t} \\
\frac{\partial v}{\partial t}
\end{array}\right)=\mathcal{L}\left(\begin{array}{l}
u(t) \\
v(t)
\end{array}\right)=\left(\begin{array}{cc}
d_{1} & d_{2} \\
-d_{3} & d_{4}
\end{array}\right)\left(\begin{array}{c}
\Delta u \\
\Delta v
\end{array}\right)+\left(\begin{array}{cc}
a-2 u-\frac{v(1+\beta v)}{(1+\alpha u+\beta v)^{2}} & -\frac{u(1+\alpha u)}{(1+\alpha u+\beta v)^{2}} \\
\frac{v^{2}}{\gamma u^{2}} & b-\frac{2 v}{\gamma u}
\end{array}\right)\left(\begin{array}{l}
u(t) \\
v(t)
\end{array}\right) .
$$

For notational simplicity, we denote

$$
\begin{aligned}
& D=\left(\begin{array}{ll}
d_{1} & d_{2} \\
-d_{3} & d_{4}
\end{array}\right), \\
& J(u, v)=\left(\begin{array}{ll}
F_{u}(u, v) & F_{v}(u, v) \\
G_{u}(u, v) & G_{v}(u, v)
\end{array}\right)=\left(\begin{array}{cc}
a-2 u-\frac{v(1+\beta v)}{(1+\alpha u+\beta v)^{2}} & -\frac{u(1+\alpha u)}{(1+\alpha u+\beta v)^{2}} \\
\frac{v^{2}}{\gamma u^{2}} & b-\frac{2 v}{\gamma u}
\end{array}\right) .
\end{aligned}
$$

In view of Theorem 5.1.1 of [10], it is obvious that $E^{*}=(u, v)$ is locally asymptotically stable when all the eigenvalues of the operator $\mathcal{L}$ possess negative real parts. On the contrary, $E^{*}=(u, v)$ is unstable. Thus the stability of the equilibrium is based on the study of the characteristic equation

$$
\operatorname{det}\left(\mu I-J_{i}\right)=\mu^{2}-\operatorname{trace} J_{i} \mu+\operatorname{det} J_{i}=0 .
$$

Here trace $J_{i}=-\lambda_{i}\left(d_{1}+d_{4}\right)+F_{u}(u, v)+G_{v}(u, v)$ and $\operatorname{det} J_{i}=\left(d_{1} d_{4}+d_{2} d_{3}\right) \lambda_{i}^{2}-\left[d_{1} G_{v}(u, v)-\right.$ $\left.d_{2} G_{u}(u, v)+d_{3} F_{v}(u, v)+d_{4} F_{u}(u, v)\right] \lambda_{i}+F_{u}(u, v) G_{v}(u, v)-F_{v}(u, v) G_{u}(u, v)$.

(1) When $E^{*}=(a, 0)$, we get

$$
J(a, 0)=\left(\begin{array}{cc}
-a & -\frac{a}{1+a \alpha} \\
0 & b
\end{array}\right)
$$

If $b>a$, then for $i=0$, we obtain trace $J_{i}=-a+b>0$. Therefore $(a, 0)$ is unstable. 
(2) When $E^{*}=\left(u^{*}, v^{*}\right)$, we have

$$
J\left(u^{*}, v^{*}\right)=\left(\begin{array}{cc}
-a+\frac{b \gamma u^{*}\left[1+(2 \alpha+b \beta \gamma) u^{*}\right]}{\left[1+(\alpha+b \beta \gamma) u^{*}\right]^{2}} & -\frac{u^{*}\left(1+\alpha u^{*}\right)}{\left[1+(\alpha+b \beta \gamma) u^{*}\right]^{2}} \\
b^{2} \gamma & -b
\end{array}\right) \triangleq\left(\begin{array}{cc}
F_{u}^{*} & F_{v}^{*} \\
G_{u}^{*} & G_{v}^{*}
\end{array}\right) .
$$

Here $-a<F_{u}^{*}<\frac{b \gamma u^{*}\left[1+(2 \alpha+b \beta \gamma) u^{*}\right]}{\left[1+(\alpha+b \beta \gamma) u^{*}\right]^{2}},-\frac{u^{*}}{1+\alpha u^{*}}<F_{v}^{*}<0, G_{u}^{*}>0, G_{v}^{*}<0$. Together with the hypothesis of (2), we can observe that

$$
F_{u}^{*}+G_{v}^{*}=-a+\frac{b \gamma u^{*}\left[1+(2 \alpha+b \beta \gamma) u^{*}\right]}{\left[1+(\alpha+b \beta \gamma) u^{*}\right]^{2}}-d<0,
$$

and then

$$
\operatorname{trace} J_{i}=-\left(d_{1}+d_{2}\right) \lambda_{i}-\left(F_{u}^{*}+G_{v}^{*}\right)<0 \text {. }
$$

In view of the hypothesis of (2), we can observe that

$$
\begin{aligned}
F_{u}^{*} G_{v}^{*}-F_{v}^{*} G_{u}^{*} & =a b-\frac{b^{2} \gamma u^{*}\left[1+(2 \alpha+b \beta \gamma) u^{*}\right]}{\left[1+(\alpha+b \beta \gamma) u^{*}\right]^{2}}+\frac{b^{2} \gamma u^{*}\left(1+\alpha u^{*}\right)}{\left[1+(\alpha+b \beta \gamma) u^{*}\right]^{2}} \\
& =a b-\frac{b^{2} \gamma u^{*}(\alpha+b \beta \gamma) u^{*}}{\left[1+(\alpha+b \beta \gamma) u^{*}\right]^{2}}>0 .
\end{aligned}
$$

We can find a sufficiently large eigenvalue $d_{2}$ to guarantee that

$$
\begin{aligned}
\operatorname{det} J_{i}= & -\left(d_{1} d_{4}+d_{2} d_{3}\right) \lambda_{i}^{2}+\left(-d_{1} G_{v}^{*}+d_{2} G_{u}^{*}-d_{3} G_{v}^{*}-d_{4} F_{u}^{*}\right) \lambda_{i} \\
& +F_{u}^{*} G_{v}^{*}-F_{v}^{*} G_{u}^{*}>0 .
\end{aligned}
$$

Thus the interior equilibrium $\left(u^{*}, v^{*}\right)$ is locally asymptotically stable.

(3) When $a+b<\frac{b \gamma u^{*}\left[1+(2 \alpha+b \beta \gamma) u^{*}\right]}{\left[1+(\alpha+b \beta \gamma) u^{*}\right]^{2}}$, we obtain

$$
\operatorname{trace} J_{i}=-a+\frac{b \gamma u^{*}\left[1+(2 \alpha+b \beta \gamma) u^{*}\right]}{\left[1+(\alpha+b \beta \gamma) u^{*}\right]^{2}}-d>0
$$

Therefore $\left(u^{*}, v^{*}\right)$ is unstable. This ends the proof of the lemma.

\section{Nonconstant positive steady states}

In this section, we offer a rigorous study of interior solutions of the corresponding strongly coupled elliptic model

$$
\begin{cases}-d_{1} \Delta u-d_{2} \Delta v=u\left(a-u-\frac{v}{1+\alpha u+\beta v}\right), & x \in \Omega, t>0, \\ d_{3} \Delta u-d_{4} \Delta v=v\left(b-\frac{v}{\gamma u}\right), & x \in \Omega, t>0, \\ \frac{\partial u}{\partial v}=\frac{\partial v}{\partial v}=0, & x \in \partial \Omega, t>0, \\ u(x, 0)=u_{0}(x)>0, \quad v(x, 0)=v_{0}(x) \geq(\not \equiv) 0, & x \in \Omega .\end{cases}
$$

Meanwhile, we will also investigate the nonconstant positive steady states of model (1.1). The existence of an interior solution of a linear cross-diffusion model has been studied by utilizing the approach proposed by [11] (upper and lower solutions) and many others. 
Lemma 3.1 Any interior solution $(u, v)$ for system (1.1) fulfills $u(x), v(x) \leq \max \{a, a b \gamma\}$ in $\bar{\Omega}$.

Proof In view of the equation of $u$ for model (3.1), we obtain

$$
-\Delta\left(d_{1} u+d_{2} v\right)=u\left(a-u-\frac{v}{1+\alpha u+\beta v}\right) \leq u(a-u) .
$$

By the maximum principle proposed by [12] we have $u(a-u) \geq 0$. Hence $u \leq a$. Together with the equation for $u$ and the equation for $v$ for system (3.1), we get

$$
-\Delta\left(-d_{3} u+d_{4} v\right)=v\left(b-\frac{v}{\gamma u}\right) \leq b v-\frac{v^{2}}{a \gamma} .
$$

Applying the maximum principle in [12], we have $b v-\frac{v^{2}}{a \gamma} \geq 0$. Thus we obtain $v \leq a b \gamma$. This ends the proof of the lemma.

Next, we introduce the compact map $C M \in C^{2}(\bar{\Omega}) \oplus C^{2}(\bar{\Omega}) \rightarrow C^{1}(\bar{\Omega}) \oplus C^{1}(\bar{\Omega})$ as follows:

$$
C M(u, v)=(-\mathcal{D} \Delta+c I)^{-1}\left(\begin{array}{c}
a u-u^{2}-\frac{u v}{1+\alpha u+\beta v}+c u \\
b v-\frac{v^{2}}{\gamma u}+c v
\end{array}\right) .
$$

Here we can choose a sufficiently large constant $c>0$ guaranteeing that the functions $a u-u^{2}-\frac{u v}{1+\alpha u+\beta v}+c u$ and $b v-\frac{v^{2}}{\gamma u}+c v$ are increasing for $u$ and $v$, respectively. We can easily observe that system (3.1) is equivalent to $(u, v)=C M(u, v)$, which implies that there exists a nonconstant interior solution of system (3.1) representing a nonconstant interior fixed point of $C M$ in $\mathcal{D}^{\prime}$. When $(a, 0)$ and $\left(u^{*}, v^{*}\right)$ exist, we suppose that they stand for an isolated fixed point of $C M$. On the contrary, there must exist a nonconstant fixed point in Int $\mathcal{D}^{\prime}$ (the interior of $\mathcal{D}^{\prime}$ ). Therefore the associate indexes in $\mathcal{Q}$ are well defined. Based on the method introduced by [13], we can directly calculate the fix point index of $C M$ with respect to $\mathcal{Q}$ over $\operatorname{Int} \mathcal{D}^{\prime}$.

Lemma 3.2 If $\frac{b \gamma u^{*}\left[1+(2 \alpha+b \beta \gamma) u^{*}\right]}{\left[1+(\alpha+b \beta \gamma) u^{*}\right]^{2}}<a+b$ and $a>\frac{b \gamma u^{*}(\alpha+b \beta \gamma) u^{*}}{\left[1+(\alpha+b \beta \gamma) u^{*}\right]^{2}}$, then $\operatorname{index}_{\mathcal{Q}}\left(C M, \operatorname{Int} \mathcal{D}^{\prime}\right)=1$.

Proof We introduce the homotopic map, for $\eta \in(0,1), C M_{\eta} \triangleq H M \in C^{2}(\bar{\Omega}) \oplus C^{2}(\bar{\Omega}) \rightarrow$ $C^{1}(\bar{\Omega}) \oplus C^{1}(\bar{\Omega})$ by

$$
H M(u, v)=(-\mathcal{D} \Delta+c I)^{-1}\left(\begin{array}{c}
a u-u^{2}-\frac{\eta u v}{1+\alpha u+\beta v}+c u \\
b v-\frac{\eta v^{2}}{\gamma u}+c v
\end{array}\right) .
$$

Utilizing a similar discussion as in Lemma 3.1, we can easily obtain that any interior fixed point $(u, v)$ for $H M$ fulfills $u(x), v(x) \leq \max \{a, a b \gamma\}$ in $\bar{\Omega}$. Thus we can easily draw a conclusion that each fixed point for $H M$ belongs to $\operatorname{Int} \mathcal{D}^{\prime}$. In addition, the corresponding index of $C M_{\eta}$ over the interior of $\mathcal{D}^{\prime}$ regarding $\mathcal{Q}$ is well defined. In view of the homotopy invariance theorem, we can obtain that index $\mathcal{Q}_{\mathcal{Q}}\left(C M_{0}, \operatorname{Int} \mathcal{D}^{\prime}\right)=\operatorname{index}_{\mathcal{Q}}\left(C M_{1}, \operatorname{Int} \mathcal{D}^{\prime}\right)=$ $\operatorname{index}_{\mathcal{S}}(B, \operatorname{Int} \mathcal{D})$, where

$$
B(u, v)=(-\mathcal{D} \Delta+c I)^{-1}\left(\begin{array}{c}
a u-u^{2}+c u \\
b v+c v
\end{array}\right) .
$$


Based on the above discussion, we argue that $\operatorname{index}_{\mathcal{S}}(B, \operatorname{Int} \mathcal{D})=1$. To prove this, we propose the homotopic map

$$
B_{\eta}(u, v)=(-\mathcal{D} \Delta+c I)^{-1}\left(\begin{array}{c}
\eta\left(a u-u^{2}\right)+c u \\
\eta b v+c v
\end{array}\right)
$$

for $\eta \in[0,1]$. Then we can directly obtain that $\operatorname{index}_{\mathcal{S}}\left(B_{0}, \operatorname{Int} \mathcal{D}\right)=\operatorname{index}_{\mathcal{S}}\left(B_{1}, \operatorname{Int} \mathcal{D}\right)$. Our concern is the eigenvalue issue

$$
B_{0}^{\prime}(0,0)(\Phi, \Psi)^{T}=\rho(\Phi, \Psi)^{T}, \quad(\Phi, \Psi) \neq(0,0)
$$

for $\rho>0$, where

$$
B_{0}(0,0)=(-\mathcal{D} \Delta+c I)^{-1}\left(\begin{array}{ll}
c & 0 \\
0 & c
\end{array}\right)
$$

The corresponding eigenfunction formulas for $\Phi$ and $\Psi$ can be performed as follows:

$$
\Phi=\sum_{k=0}^{\infty} \sum_{j=1}^{\mathcal{M}_{k}} \phi_{k j} \mathcal{E}_{k j} \quad \text { and } \quad \Psi=\sum_{k=0}^{\infty} \sum_{j=1}^{\mathcal{M}_{k}} \psi_{k j} \mathcal{E}_{k j}
$$

where $\phi_{k j}, \psi_{k j} \in \mathbb{R}$. We easily rewrite system (3.2) as follows:

$$
\left\{\begin{array}{l}
\sum_{k=0}^{\infty} \sum_{j=1}^{\mathcal{M}_{k}}\left[\left(d_{1} \lambda_{k} \rho+c \rho-c\right) \phi_{k j}+d_{2} \lambda_{k} \rho \psi_{k j}\right] \mathcal{E}_{k j}=0, \\
\sum_{k=0}^{\infty} \sum_{j=1}^{\mathcal{M}_{k}}\left[-d_{3} \lambda_{k} \rho \phi_{k j}+\left(d_{4} \lambda_{k} \rho+c \rho-c\right) \psi_{k j}\right] \mathcal{E}_{k j}=0 .
\end{array}\right.
$$

As we know, $\left\{\mathcal{E}_{k j}: 1 \leq j \leq \mathcal{M}_{k}\right\}$ in $L^{2}(\Omega)$ stands for a complete orthonormal basis. By multiplying the above two equations by $\varphi_{k j}$ and integrating over $\Omega$ we get the equation

$$
\left(\begin{array}{cc}
d_{1} \lambda_{k} \rho+c \rho-c & d_{2} \lambda_{k} \rho \\
-d_{3} \lambda_{k} \rho & d_{4} \lambda_{k} \rho+c \rho-c
\end{array}\right)\left(\begin{array}{l}
\phi_{k j} \\
\psi_{k j}
\end{array}\right)=0 .
$$

Therefore

$$
\begin{aligned}
& \operatorname{det}\left(\begin{array}{cc}
d_{1} \lambda_{k} \rho+c \rho-c & d_{2} \lambda_{k} \rho \\
-d_{3} \lambda_{k} \rho & d_{4} \lambda_{k} \rho+c \rho-c
\end{array}\right) \\
& \quad=\left[\left(d_{1} \lambda_{k}+c\right)\left(d_{4} \lambda_{k}+c\right)+d_{2} d_{3} \lambda_{k}^{2}\right] \rho^{2}-c\left(d_{1} \lambda_{k}+d_{4} \lambda_{k}+2 c\right) \rho+c^{2} \\
& \quad=0 .
\end{aligned}
$$

\section{Denoting}

$$
\rho_{ \pm}=\frac{\bar{\Lambda} \pm \sqrt{\bar{\Lambda}^{2}-4\left[\left(d_{1} \lambda_{k}+c\right)\left(d_{4} \lambda_{k}+c\right)+d_{2} d_{3} \lambda_{k}^{2}\right] c^{2}}}{2\left[\left(d_{1} \lambda_{k}+c\right)\left(d_{4} \lambda_{k}+c\right)+d_{2} d_{3} \lambda_{k}^{2}\right]},
$$


where $\bar{\Lambda}=c\left(d_{1} \lambda_{k}+d_{4} \lambda_{k}+2 c\right)$, we have

$$
\begin{aligned}
\rho_{+}< & \frac{\bar{\Lambda}+\sqrt{\bar{\Lambda}^{2}-4\left(d_{1} \lambda_{k}+c\right)\left(d_{4} \lambda_{k}+c\right) c^{2}}}{2\left(d_{1} \lambda_{k}+c\right)\left(d_{4} \lambda_{k}+c\right)} \\
& =\frac{c\left[\left(d_{1} \lambda_{k}+c\right)+\left(d_{4} \lambda_{k}+c\right)+\left|\left(d_{1} \lambda_{k}+c\right)-\left(d_{4} \lambda_{k}+c\right)\right|\right]}{2\left(d_{1} \lambda_{k}+c\right)\left(d_{4} \lambda_{k}+c\right)} \leq 1 .
\end{aligned}
$$

Based on Lemma 13.1 of [14], we get $\operatorname{index}_{\mathcal{S}}\left(B_{0}, \operatorname{Int} \mathcal{D}\right)=1$. Hence $\operatorname{index}_{\mathcal{S}}(B, \operatorname{Int} \mathcal{D})=$ $\operatorname{index}_{\mathcal{S}}\left(B_{1}, \operatorname{Int} \mathcal{D}\right)=1$. This finishes the proof of the lemma.

Lemma 3.3 $\operatorname{index}_{\mathcal{Q}}(C M,(a, 0))=0$.

Proof Denote $\overline{\mathcal{Q}}_{(a, 0)}=C_{v}^{1}(\bar{\Omega}) \oplus \mathcal{S}, \mathcal{W}_{(a, 0)}=C_{v}^{1}(\bar{\Omega}) \oplus\{0\}$, and

$$
C M^{\prime}(a, 0)=(-\mathcal{D} \Delta+c I)^{-1}\left(\begin{array}{cc}
c-a & -\frac{a}{1+a \alpha} \\
0 & b+c
\end{array}\right) .
$$

Set $C M^{\prime}(a, 0)(\Phi, \Psi)^{T}=(\Phi, \Psi)^{T} \in \overline{\mathcal{Q}}_{(a, 0)}$. Then we get

$$
\begin{cases}d_{1} \Delta \Phi+d_{2} \Delta \Psi=a \Phi+\frac{a}{1+a \alpha} \Psi & \text { in } \Omega, \\ d_{3} \Delta \Phi-d_{4} \Delta \Psi=b \Psi & \text { in } \Omega, \\ \frac{\partial \Phi}{\partial \nu}=\frac{\partial \Psi}{\partial \nu}=0 & \text { on } \partial \Omega .\end{cases}
$$

From the first equation of system (3.4) we have $\Phi=\Psi=0$ in $\Omega$. Thus $I-C M^{\prime}(a, 0)$ represents an invertible matrix on $\overline{\mathcal{Q}}_{(a, 0)}$. On the other hand, we can easily check that $C M^{\prime}(a, 0)$ possesses property $\gamma$, which means that for $(\Phi, \Psi) \equiv(0,1) \in \overline{\mathcal{Q}}_{(a, 0)} \backslash \mathcal{W}_{(a, 0)}$ and $s=\frac{c}{b+c} \in(0,1),\left[(\Phi, \Psi)^{T}-s C M^{\prime}(a, 0)(\Phi, \Psi)^{T}\right] \in \mathcal{W}_{(a, 0)}$. Hence, applying Lemma 4.1(i) in [13], we obtain a precise result. This finishes the proof of the lemma.

Denote

$$
\begin{aligned}
\operatorname{Det}(\lambda) & :=\operatorname{det}\left(\begin{array}{cc}
d_{1} \lambda-F_{u}^{*} & d_{2} \lambda-F_{v}^{*} \\
-d_{3} \lambda-G_{u}^{*} & d_{4} \lambda-G_{v}^{*}
\end{array}\right) \\
& =\left(d_{1} d_{4}+d_{2} d_{3}\right) \lambda^{2}-\left[d_{1} G_{v}^{*}-d_{2} G_{u}^{*}+d_{3} F_{v}^{*}+d_{4} F_{u}^{*}\right] \lambda+F_{u}^{*} G_{v}^{*}-F_{v}^{*} G_{u}^{*} .
\end{aligned}
$$

In the rest of the paper, we consider index $\mathcal{Q}_{\mathcal{Q}}\left(C M,\left(u^{*}, v^{*}\right)\right)$ in the following three situations: (1) For all $\lambda>0$, $\operatorname{Det}(\lambda)>0$; (2) There exists, with multiplicity one, precisely a simple interior solution for $\operatorname{Det}(\lambda)=0$; (3) There exist, with multiplicity one, two interior solutions for $\operatorname{Det}(\lambda)=0$. Now we present our main theoretical result.

\section{Theorem 3.1}

(1) If $\operatorname{Det}(\lambda)>0$ for all $\lambda>0$, then $\operatorname{index}_{\mathcal{Q}}\left(C M,\left(u^{*}, v^{*}\right)\right)=1$;

(2) If $\operatorname{Det}(\lambda)=0$ has exactly a multiplicity-one simple positive solution $\lambda^{*}$ in an open interval $\left(\lambda_{k^{*}}, \lambda_{k^{*}+1}\right)$ for some nonnegative integer $k^{*}$, then

$$
\operatorname{index}_{\mathcal{Q}}\left(C M,\left(u^{*}, v^{*}\right)\right)= \begin{cases}-1, & \sum_{k=0}^{k^{*}} \mathcal{M}_{k} \text { is odd } \\ 1, & \sum_{k=0}^{k^{*}} \mathcal{M}_{k} \text { is even }\end{cases}
$$


In addition, when $\sum_{k=0}^{k^{*}} \mathcal{M}_{k}$ is odd, system (3.1) possesses at least one nonconstant interior solution;

(3) Suppose that $\operatorname{Det}(\lambda)=0$ has two interior solutions $\lambda_{+}^{*}$ and $\lambda_{-}^{*}$ in two open intervals $\left(\lambda_{k_{1}^{*}}, \lambda_{k_{1}^{*}+1}\right)$ and $\left(\lambda_{k_{2}^{*}}, \lambda_{k_{2}^{*}+1}\right)$, respectively, where $k_{1}^{*}>k_{2}^{*} \geq 0$. Then

$$
\operatorname{index}_{\mathcal{Q}}\left(C M,\left(u^{*}, v^{*}\right)\right)= \begin{cases}-1, & \sum_{k=k_{1}^{*}+1}^{k_{2}^{*}} \mathcal{M}_{k} \text { is odd }, \\ 1, & \sum_{k=k_{1}^{*}+1}^{k_{2}^{*}} \mathcal{M}_{k} \text { is even } .\end{cases}
$$

In addition, when $\sum_{k=k_{1}^{*}+1}^{k_{2}^{*}} \mathcal{M}_{k}$ is odd, system (3.1) possesses at least one nonconstant interior solution;

(4) Suppose that $a<\frac{b \gamma u^{*}\left[1+(2 \alpha+b \beta \gamma) u^{*}\right]}{\left[1+(\alpha+b \beta \gamma) u^{*}\right]^{2}}$ and $\frac{F_{u}^{*}}{d_{1}} \in\left(\lambda_{k^{*}}, \lambda_{k^{*}+1}\right)$ for some nonnegative integer $k^{*}$. Then there is a constant $d_{4}^{\prime}>0$ such that for $d_{4}^{\prime}<d_{4}$,

$$
\operatorname{index}_{\mathcal{Q}}\left(C M,\left(u^{*}, v^{*}\right)\right)= \begin{cases}-1, & \sum_{k=0}^{k^{*}} \mathcal{M}_{k} \text { is odd } \\ 1, & \sum_{k=0}^{k^{*}} \mathcal{M}_{k} \text { is even }\end{cases}
$$

In addition, when $\sum_{k=0}^{k^{*}} \mathcal{M}_{k}$ is odd, system (3.1) possesses at least one nonconstant interior solution.

Proof By applying the elliptic PDE theory and the eigenfunction theory, we prove that $\operatorname{index}_{\mathcal{Q}}\left(C M,\left(u^{*}, v^{*}\right)\right)=1$.

(1) First, note that $\overline{\mathcal{Q}}_{\left(u^{*}, v^{*}\right)}=\mathcal{W}_{\left(u^{*}, v^{*}\right)}=\mathcal{A}$ and

$$
C M^{\prime}\left(u^{*}, v^{*}\right)=(-\mathcal{D} \Delta+c I)^{-1}\left(\begin{array}{cc}
c+F_{u}^{*} & F_{v}^{*} \\
G_{u}^{*} & c+G_{v}^{*}
\end{array}\right)
$$

Set $C M^{\prime}\left(u^{*}, v^{*}\right)(\Phi, \Psi)^{T}=(\Phi, \Psi)^{T} \in \overline{\mathcal{Q}}_{\left(u^{*}, v^{*}\right)}$. Using the corresponding eigenfunction formulas in Lemma 3.2 for $\Phi$ and $\Psi$, we get

$$
\left(\begin{array}{cc}
d_{1} \lambda_{k}-F_{u}^{*} & -d_{2} \lambda_{k}-F_{v}^{*} \\
d_{3} \lambda_{k}-G_{u}^{*} & d_{4} \lambda_{k}-G_{v}^{*}
\end{array}\right)\left(\begin{array}{l}
\phi_{k j} \\
\psi_{k j}
\end{array}\right)=0 .
$$

Hence $\operatorname{Det}\left(\lambda_{k}\right)=\left(d_{1} d_{4}+d_{2} d_{3}\right) \lambda_{k}^{2}-\left[d_{1} G_{v}^{*}-d_{2} G_{u}^{*}+d_{3} F_{v}^{*}+d_{4} F_{u}^{*}\right] \lambda_{k}+F_{u}^{*} G_{v}^{*}-F_{v}^{*} G_{u}^{*}>0$ since $\operatorname{Det}(\lambda)>0$. This implies that $I-C M^{\prime}\left(u^{*}, v^{*}\right)$ is an invertible matrix on $\overline{\mathcal{Q}}_{\left(u^{*}, v^{*}\right)}$. Therefore $C M^{\prime}\left(u^{*}, v^{*}\right)$ does not possess property $\gamma$ on $\overline{\mathcal{Q}}_{\left(u^{*}, v^{*}\right)}$.

Now we offer a rigorous proof to guarantee that $\delta=0$. Here $\delta$ is described in Lemma 4.1 of [13]. For $\rho>0$, we study the eigenvalue issue $\left(C M^{\prime}\left(u^{*}, v^{*}\right)-I\right)(\Phi, \Psi)^{T}=\rho(\Phi, \Psi)^{T}$, $(\Phi, \Psi) \neq(0,0)$, which means that for some positive constant $\rho$, the model

$$
\begin{cases}(\rho+1)\left(-d_{1} \Delta \Phi-d_{2} \Delta \Psi\right)=\left(F_{u}^{*}-\rho c\right) \Phi+F_{v}^{*} \Psi & \text { in } \Omega, \\ (\rho+1)\left(d_{3} \Delta \Phi-d_{4} \Delta \Psi\right)=G_{u}^{*} \Phi+\left(G_{v}^{*}-\rho c\right) \Psi & \text { in } \Omega, \\ \frac{\partial \Phi}{\partial v}=\frac{\partial \Psi}{\partial v}=0 & \text { on } \partial \Omega \\ \Phi \Psi \neq 0 & \text { in } \Omega,\end{cases}
$$


has a nontrivial solution if and only if $\rho$ satisfies the quadratic equation $\operatorname{Det}\left(Q\left(\rho, \lambda_{k}\right)\right)=0$, where

$$
\begin{aligned}
\operatorname{Det}\left(Q\left(\rho, \lambda_{k}\right)\right):= & \operatorname{det}\left(\begin{array}{cc}
(\rho+1) d_{1} \lambda_{k}+\rho c-F_{u}^{*} & (\rho+1) d_{2} \lambda_{k}-F_{v}^{*} \\
-(\rho+1) d_{3} \lambda_{k}-G_{u}^{*} & (\rho+1) d_{4} \lambda_{k}+\rho c-G_{v}^{*}
\end{array}\right) \\
= & {\left.\left[d_{1} \lambda_{k}+c\right)\left(d_{4} \lambda_{k}+c\right)+d_{2} d_{3} \lambda_{k}^{2}\right] \rho^{2}+2\left(d_{1} d_{4}+d_{2} d_{3}\right) \lambda_{k}^{2} \rho } \\
& +\left[\left(-d_{1} G_{v}^{*}-d_{2} G_{u}^{*}+d_{3} F_{v}^{*}-d_{4} F_{u}^{*}\right) \lambda_{k}+\left(d_{1} \lambda_{k}-F_{u}^{*}\right) c\right. \\
& \left.+\left(d_{4} \lambda_{k}-G_{v}^{*}\right) c\right] \rho+\operatorname{Det}\left(\lambda_{k}\right) .
\end{aligned}
$$

For $\lambda_{k}>0$, we obtain $\operatorname{Det}\left(\lambda_{k}\right)>0$. This implies that $\operatorname{Det}\left(Q\left(\rho, \lambda_{k}\right)\right)=0$ may have either precisely a multiplicity-two simple interior solution, or two multiplicity-one interior solutions, or no interior solution. Thus we can conclude that for $k \geq 0$, the total algebraic multiplicity for $\operatorname{Det}\left(Q\left(\rho, \lambda_{k}\right)\right)=0$ equals zero or two, which means that $\delta=0$. Finally, we obtain that index $\operatorname{x}_{\mathcal{Q}}\left(C M,\left(u^{*}, v^{*}\right)\right)=1$.

(2) In view of the assumption introduced by (2), we can observe that for a nonnegative integer $k$, $\operatorname{Det}\left(\lambda_{k}\right) \neq 0$. Clearly, $I-C M^{\prime}\left(u^{*}, v^{*}\right)$ is an invertible matrix on $\overline{\mathcal{Q}}_{\left(u^{*}, v^{*}\right)}$. Meanwhile, we can conclude that $C M^{\prime}\left(u^{*}, v^{*}\right)$ cannot possess property $\gamma$ on $\overline{\mathcal{Q}}_{\left(u^{*}, v^{*}\right)}$. Next, we focus on the total algebraic multiplicity $\delta$ for each eigenvalue of $C M^{\prime}\left(u^{*}, v^{*}\right)-I$, which is greater than zero. When $\lambda_{0}=0(k=0)$, we $\operatorname{get} \operatorname{Det}(Q(\rho, 0))=c^{2} \rho^{2}-c\left(F_{u}^{*}+G_{v}^{*}\right) \rho+F_{u}^{*} G_{v}^{*}-F_{v}^{*} G_{u}^{*}=0$. By the hypothesis of (2) we get $F_{u}^{*} G_{v}^{*}-F_{v}^{*} G_{u}^{*} \leq 0$. Obviously, $\operatorname{Det}(Q(\rho, 0))=0$ has exactly a simple interior solution. Together with $1 \leq k \leq k^{*}$ and the hypothesis in (2), wee get $\operatorname{Det}\left(\lambda_{k}\right)<0$, and hence $\operatorname{Det}\left(Q\left(\rho, \lambda_{k}\right)\right)=0$ possesses precisely one positive simple solution. If $k \geq k^{*}+1$, then $\operatorname{Det}\left(\lambda_{k}\right)>0$, and hence $\operatorname{Det}\left(Q\left(\rho, \lambda_{k}\right)\right)=0$ may have either precisely one multiplicity-two simple interior solution, or two multiplicity-one interior solutions, or no interior solution. This discussion yields that $\sum_{k=0}^{k^{*}} \mathcal{M}_{k}+t=\delta$, where $t$ is an even number or 0 . In addition, suppose on the contrary that model (3.1) possesses no nonconstant interior solution. By Lemma 3.2, Lemma 3.3, and the last discussion we get

$$
\begin{aligned}
1 & =\operatorname{index}_{\mathcal{Q}}\left(C M, \operatorname{Int} \mathcal{D}^{\prime}\right)=\operatorname{index}_{\mathcal{Q}}(C M,(a, 0))+\operatorname{index}_{\mathcal{Q}}\left(C M,\left(u^{*}, v^{*}\right)\right) \\
& =0+(-1)=-1
\end{aligned}
$$

This contradiction tells us that system (3.1) possesses at least one nonconstant interior solution. The above argument derives the ideal outcome.

(3) The proof of this part is similar to that of part (2), and we omit it.

(4) Due to $a<\frac{b \gamma u^{*}\left[1+(2 \alpha+b \beta \gamma) u^{*}\right]}{\left[1+(\alpha+b \beta \gamma) u^{*}\right]^{2}}$, we get $F_{u}^{*}>0$. Let $\operatorname{Det}(\lambda)=0$, which means that

$$
\operatorname{Det}(\lambda)=\left(d_{1} d_{4}+d_{2} d_{3}\right) \lambda^{2}-\Theta \lambda+F_{u}^{*} G_{v}^{*}-F_{v}^{*} G_{u}^{*}=0,
$$

where

$$
\Theta=d_{1} G_{v}^{*}-d_{2} G_{u}^{*}+d_{3} F_{v}^{*}+d_{4} F_{u}^{*}
$$

By direct calculation we get that

$$
\lim _{d_{4} \rightarrow \infty} \lambda_{+}^{*}=\lim _{d_{1} \rightarrow \infty} \frac{\Theta+\sqrt{\Theta^{2}-4\left(d_{1} d_{4}+d_{2} d_{3}\right)\left(F_{u}^{*} G_{v}^{*}-F_{v}^{*} G_{u}^{*}\right)}}{2\left(d_{1} d_{4}+d_{2} d_{3}\right)}=\frac{F_{u}^{*}}{d_{1}},
$$




$$
\lim _{d_{4} \rightarrow \infty} \lambda_{-}^{*}=\lim _{d_{1} \rightarrow \infty} \frac{\Theta-\sqrt{\Theta^{2}-4\left(d_{1} d_{4}+d_{2} d_{3}\right)\left(F_{u}^{*} G_{v}^{*}-F_{v}^{*} G_{u}^{*}\right)}}{2\left(d_{1} d_{4}+d_{2} d_{3}\right)}=0 .
$$

Hence we can find a constant $d_{4}^{\prime}>0$ guaranteeing $\lambda_{+}^{*}>\lambda_{k^{*}}$ and $\lambda_{-}^{*}<\lambda_{1}$ if $d_{4}^{\prime}<d_{4}$ due to $\frac{F_{u}^{*}}{d_{1}} \in\left(\lambda_{k^{*}}, \lambda_{k^{*}+1}\right)$. Therefore, for a nonnegative integer $k$, $\operatorname{Det}\left(\lambda_{k}\right) \neq 0$. We can observe that $I-C M^{\prime}\left(u^{*}, v^{*}\right)$ is an invertible matrix on $\overline{\mathcal{Q}}_{\left(u^{*}, v^{*}\right)}$. Meanwhile, we can conclude that $C M^{\prime}\left(u^{*}, v^{*}\right)$ cannot possess property $\gamma$ on $\overline{\mathcal{Q}}_{\left(u^{*}, v^{*}\right)}$. On the other hand, note that $\operatorname{Det}\left(\lambda_{1}\right)<0$ when

$$
d_{4}>\sigma_{1}:=\frac{d_{2} d_{3} \lambda_{1}^{2}+\left(-d_{1} G_{v}^{*}+d_{2} G_{u}^{*}-d_{3} F_{v}^{*}\right) \lambda_{1} F_{u}^{*} G_{v}^{*}-F_{v}^{*} G_{u}^{*}}{F_{u}^{*} \lambda_{1}-d_{1} \lambda_{1}^{2}}
$$

and $\operatorname{Det}\left(\lambda_{k^{*}}\right)<0$ when

$$
d_{4}>\sigma_{2}:=\frac{d_{2} d_{3} \lambda_{k^{*}}^{2}+\left(-d_{1} G_{v}^{*}+d_{2} G_{u}^{*}-d_{3} F_{v}^{*}\right) \lambda_{k^{*}} F_{u}^{*} G_{v}^{*}-F_{v}^{*} G_{u}^{*}}{F_{u}^{*} \lambda_{k^{*}}-d_{1} \lambda_{k^{*}}^{2}} .
$$

We can take a constant $d_{4}^{\prime}>\max \left\{\sigma_{1}, \sigma_{2}\right\}$. We easily verify that for each $1 \leq k \leq k^{*}$, $\operatorname{Det}\left(Q\left(\rho, \lambda_{k}\right)\right)=0$ has exactly one simple interior solution due to $\operatorname{Det}\left(\lambda_{k}\right)<0$. Meanwhile, together with $k \geq 1+k^{*}$ and $\operatorname{Det}\left(\lambda_{k}\right)>0$, $\operatorname{Det}\left(Q\left(\rho, \lambda_{k}\right)\right)=0$ has either precisely one multiplicity-two simple interior solution, or two multiplicity-one interior solutions, or no interior solution. According to the above discussion, we derive that $\sum_{k=0}^{k^{*}} \mathcal{M}_{k}+0=\delta$. In addition, suppose on the contrary that model (3.1) possesses no nonconstant interior solution. By Lemmas 3.2 and 3.3 and the last argument we get

$$
\begin{aligned}
1 & =\operatorname{index}_{\mathcal{Q}}\left(C M, \operatorname{Int} \mathcal{D}^{\prime}\right)=\operatorname{index}_{\mathcal{Q}}(C M,(a, 0))+\operatorname{index}_{\mathcal{Q}}\left(C M,\left(u^{*}, v^{*}\right)\right) \\
& =0+(-1)=-1
\end{aligned}
$$

This contradiction tells us that system (3.1) possesses at least one nonconstant interior solution. The above argument derives the ideal outcome. This ends the proof of Theorem 3.1.

Example 1 Choosing $a=2, b=2, \alpha=1, \beta=1$, and $\gamma=2$, we obtain $\left(u^{*}, v^{*}\right)=\left(\frac{5+\sqrt{65}}{10}\right.$, $\left.\frac{10+2 \sqrt{65}}{5}\right) \approx(1.3062,5.2249)$. Thus $\frac{b \gamma u^{*}\left[1+(2 \alpha+b \beta \gamma) u^{*}\right]}{\left[1+(\alpha+b \beta \gamma) u^{*}\right]^{2}} \approx 0.8141<a+b=4$ and $\left.a=2\right\rangle$ $\frac{b \gamma u^{*}(\alpha+b \beta \gamma) u^{*}}{\left[1+(\alpha+b \beta \gamma) u^{*}\right]^{2}} \approx 0.6017$. Therefore system (1.1) possesses at least one nonconstant interior solution.

\section{Conclusion}

In this paper, we introduce a complicated predator-prey linear cross-diffusion system with the Beddington-DeAngelis and Tanner functional response and applied an effective rigorous approach to obtain the existence of nonconstant positive steady state of the corresponding system. By considering $\operatorname{Det}(\lambda)$ in three situations we can easily compute index $\mathcal{Q}\left(C M,\left(u^{*}, v^{*}\right)\right)$. We proposed some parameter conditions to guarantee the local stability of the unique interior equilibrium.

It is worth noting that our methods in this paper can be applied to investigation of a class of nonlinear prey-taxis model with more general functional responses. By utilizing the fixed point index theory to get the existence of nonconstant interior steady states it is necessary to provide an a priori estimate and regularity of steady states, which plays an important role (see [15]). 


\section{Acknowledgements}

The author would like to thank the anonymous referee for constructive comments, which led to an improvements of this paper.

Funding

This work was supported in part by the National Natural Science Foundation of China (No. 11671406).

\section{Abbreviations}

Not applicable.

\section{Availability of data and materials}

Not applicable.

\section{Competing interests}

The author declares that they have no competing interests.

\section{Authors' contributions}

The author read and approved the final manuscript.

\section{Publisher's Note}

Springer Nature remains neutral with regard to jurisdictional claims in published maps and institutional affiliations.

Received: 7 July 2020 Accepted: 20 November 2020 Published online: 07 January 2021

\section{References}

1. Luo, D.: The study of global stability of a periodic Beddington-DeAngelis and Tanner predator-prey model. Results Math. 101, 1-18 (2019)

2. Liu, N.W., Li, N.: Global stability of a predator-prey model with Beddington-DeAngelis and Tanner functional response. Electron. J. Qual. Theory Differ. Equ. 2017, 35 (2017)

3. Shi, J., Xie, Z., Little, K.: Cross-diffusion induced instability and stability in reaction-diffusion systems. J. Appl. Anal. Comput. 1(1), 95-119(2011)

4. Farkas, M.: Two ways of modelling cross-diffusion. Nonlinear Anal. 30(2), 1225-1233 (1997)

5. Gambino, G., Lombardo, M.C., Lupo, S., Sammartino, M.: Super-critical and sub-critical bifurcations in a reaction-diffusion Schnakenberg model with linear cross-diffusion. Ric. Mat. 65, 449-467 (2016)

6. Beddington, J.R.: Mutual interference between parasites or predators and its effect on searching efficiency. J. Anim. Ecol. 44, 331-340 (1975)

7. DeAngelis, D.L., Goldstein, R.A., O'Neill, R.V.: A model for trophic interaction. Ecology 56, 881-892 (1975)

8. Gala, S., Liu, Q., Ragusa, M.A.: A new regularity criterion for the nematic liquid crystal flows. Appl. Anal. 91(9), $1741-1747$ (2012)

9. Benbernou, S., Gala, S., Ragusa, M.A.: On the regularity criteria for the 3D magnetohydrodynamic equations via two components in terms of BMO space. Math. Methods Appl. Sci. 37(15), 2320-2325 (2014)

10. Henry, D.: Geometric Theory of Semilinear Parabolic Equations. Lecture Notes in Math. Springer, Berlin (1981)

11. Ko, W., Ryu, K.: On a predator-prey system with cross diffusion representing the tendency of predators in the presence of prey species. J. Math. Anal. Appl. 341, 1133-1142 (2008)

12. Lou, Y., Ni, W.: Diffusion, self-diffusion and cross-diffusion. J. Differ. Equ. 131, 79-131 (1996)

13. Li, C., Wang, X., Shao, Y.: Steady states of a predator-prey model with prey-taxis. Nonlinear Anal. 97, 155-168 (2014)

14. Amann, H.: Fixed point and nonlinear eigenvalue problems in ordered Banach spaces. SIAM Rev. 18, 620-709 (1976)

15. Zhang, L., Fu, S.: Global bifurcation for Holling-Tanner predator-prey model with prey taxis. Nonlinear Anal., Real World Appl. 47, 460-472 (2019)

\section{Submit your manuscript to a SpringerOpen ${ }^{\circ}$ journal and benefit from:}

- Convenient online submission

- Rigorous peer review

- Open access: articles freely available online

- High visibility within the field

- Retaining the copyright to your article

Submit your next manuscript at $\boldsymbol{~ s p r i n g e r o p e n . c o m ~}$ 